\title{
Fabrication of hollow PbS nanospheres and application in phenol release
}

\author{
Jian Ye*, Lanping Sun and Shengping Gao
}

\begin{abstract}
This article demonstrates a versatile method to prepare the hollow PbS nanospheres via the template method. First, the latex poly (vinyl benzyl chloride) (PVBC) nanoparticles were synthesized by the radical polymerization, followed by the atom transfer reversible polymerization of lead (II) dimethacrylate (Pb $\left.(\mathrm{MA})_{2}\right)$ on the surfaces of the latex nanoparticles. Then, the ethanethioamide was reacted with the nanoparticles to afford the PbS. By calcination at $600^{\circ} \mathrm{C}$ for $6 \mathrm{~h}$, the template was removed to obtain the hollow PbS nanospheres. The structure, morphology and optical properties of the hollow PbS nanospheres were carefully investigated. The received hollow PbS nanospheres could be used for the controlled release of phenol after absorbing phenol solution.
\end{abstract}

Keywords: Hollow PbS nanospheres; Lead dimethacrylate; ATRP; Release

\section{Introduction}

Semiconductor materials have been the focus in material science, due to their prominent optical and electronic properties and their potential applications for the preparation of optical signal processors and switches (Ridley et al. 1999). As an important IV-VI group semiconductor, lead sulfide $(\mathrm{PbS})$ has attracted considerable attention for many decades due to its specially small band gap $(0.41 \mathrm{eV}$ at $300 \mathrm{~K})$ and a larger exciton Bohr radius of $18 \mathrm{~nm}$ (Machol et al. 1993). What is more, a blue shift from near infrared to visible region can occur. This kind of material presented fantastic optical and electric properties, which could be potentially applied widely in optoelectronic materials such as inductor, infrared detector, photoelectric converter and solar cells (McDonald et al. 2005; Deng et al. 2009; Gao et al. 2011; Lee et al. 2008; Noone et al. 2010; Asunskis et al. 2008; Jain et al. 2010). The design and fabrication of $\mathrm{PbS}$ nanostructure have attracted considerable attention in recent years. The synthesis of closed nanorods (Wang \& Yang 2000), nanowires (Lau et al. 2009) and dendritic structures (Zhou et al. 2006) by different methods such as solvothermal, microwave irradiation and thermal decomposition have been reported by many groups. Additionally, $\mathrm{PbS}$ crystals with other morphologies have also been reported (Lee et al. 2002; Peng et al. 2008).

\footnotetext{
* Correspondence: bbxyyj@126.com

Department of Chemical and Environmental Engineering, Bengbu College, Bengbu 233030, China
}

Recently, hollow micro- or nanospheres have attracted much attention because of their specific structures and potential applications. Owing to their low density, large surface area, and surface permeability, hollow spheres were widely used as artificial cell, catalysts, fillers, and capsules for controlled release of drugs and dyes (Huang et al. 1999; Caruso 2000). The hollow nanospheres could be often prepared via the template methods, such as the one-pot preparation of hollow silica spheres by using thermosensitive poly ( $\mathrm{N}$-isopropylacrylamide) as a reversible template (Du et al. 2009), and the fabrication of polymer nanocapsules with cross-linked organic-inorganic hybrid walls (Chen et al. 2012). The hollow PbS nanospheres have been obtained via the sonochemical synthesis method (Wang et al. 2006) and the block copolymer microemulsion based approach (Ding et al. 2007; Gao et al. 2005). However, few articles reported on the synthesis of hollow $\mathrm{PbS}$ nanospheres prepared with the assistance of any surfactant and atom transfer reversible polymerization (ATRP) process. Furthermore, the application of hollow $\mathrm{PbS}$ nanospheres in the field of controlled release needs to be explored.

In this paper, a simple chemical precipitation route was demonstrated for the fabrication of poly (vinyl benzyl chloride)@PbS (PVBC@PbS) core-shell and hollow $\mathrm{PbS}$ nanospheres. The structure, morphology and optical properties of the hollow nanospheres were investigated. The hollow $\mathrm{PbS}$ nanospheres embedded with phenol

\section{Springer}

(C) 2013 Ye et al.; licensee Springer. This is an Open Access article distributed under the terms of the Creative Commons Attribution License (http://creativecommons.org/licenses/by/2.0), which permits unrestricted use, distribution, and reproduction in any medium, provided the original work is properly cited. 
could be used for the controlled release of phenol. It is worth noting that the method demonstrated in this article may potentially find useful application in the field of controlled release.

\section{Experimental work Materials}

The vinyl benzyl chloride (VBC), polyethylene-polypropylene glycol, sodium dodecylbenzenesulphonate, azobisisobutyronitrile (AIBN) and ethanethioamide were purchased from Sinopharm Chemical Reagent Corporation Ltd. The $p$-toluenesulfonylchloride and 2, 2'-dipyridyl (Bpy, >99\%) were purchased from ACROS. The solvents were purchased from Tianjin Fuchen Chemical Reagent Factory. The chemicals were used as received without further purification. Copper (I) chloride $(\mathrm{CuCl})$ was purified according to procedures described in the literature (Matyjaszewski et al. 1999). Lead dimethacrylate (LDMA) was synthesized according to the literature (Dave 1984) and re-crystallized from ethanol.

\section{Preparation of poly (vinyl benzyl chloride) (PVBC) latex nanospheres}

Vinyl benzyl chloride $(21.4 \mathrm{~g}, 0.14 \mathrm{~mol})$, polyethylenepolypropylene glycol $(4.0 \mathrm{~g})$, sodium dodecylbenzenesulphonate $(0.5 \mathrm{~g}, 1.43 \mathrm{mmol})$ and deionized water $(74.0 \mathrm{~mL})$ were charged into a $250 \mathrm{~mL}$ flask. After vigorous stirring for $30 \mathrm{~min}$, the AIBN (0.065 g, $0.39 \mathrm{mmol})$ was added and the reaction mixture was heated to $65^{\circ} \mathrm{C}$ and stirred for $10 \mathrm{~h}$. Then, the mixture was poured into a $500 \mathrm{~mL}$ beaker containing ethanol. The precipitated polymer was filtered and extracted with ethanol and deionized $\mathrm{H}_{2} \mathrm{O}$ for $6 \mathrm{~h}$. The PVBC nanospheres were dried in vacuum at $80^{\circ} \mathrm{C}$ for $12 \mathrm{~h}$ and isolated as white solid in a weight yield of $87 \%$, and the result could be reproduced by the careful control of the reaction conditions.

\section{Surface-initiated atom transfer radical polymerization}

Into a Pyrex tube, the PVBC latex nanospheres $(0.04 \mathrm{~g})$ were dispersed in $40 \mathrm{~mL}$ of ethanol solution containing LDMA (3.2 g, $8.5 \mathrm{mmol}$ ) and $p$-toluenesulfonylchloride ( $64.8 \mathrm{mg}, 0.34 \mathrm{mmol}$ ). The mixture was purged by nitrogen for $30 \mathrm{~min}$, followed by the addition of $\mathrm{CuCl}(33.7 \mathrm{mg}$, $0.34 \mathrm{mmol})$ and Bpy (106.2 $\mathrm{mg}, 0.34 \mathrm{mmol})$. The $\mathrm{CuCl}$ and Bpy were used as the catalyst in the atom transfer radical polymerization. The Pyrex tube was sealed and kept at $90^{\circ} \mathrm{C}$ for several hours after purging with argon for another $10 \mathrm{~min}$. The poly (vinyl benzyl chloride) grafted poly (lead dimethacrylate) (PVBC-g-PLDMA) nanospheres were collected by centrifuging at $20000 \mathrm{rpm}$. The collected nanospheres were re-dispersed in ethanol and centrifuged at $1000 \mathrm{rpm}$ for $5 \mathrm{~min}$ to remove any $\mathrm{Cu}$ (II) precipitate formed in the ATRP process. The nanospheres were then collected by centrifuging at $20000 \mathrm{rpm}$ and subjected to repeated cycles of washing with ethanol and centrifugation to remove the untreated monomer and homo-polymer before further characterization.

\section{Reaction with ethanethioamide}

The PVBC-g-PLDMA nanospheres were dispersed into $10 \mathrm{~mL}$ ethanol in a $100 \mathrm{~mL}$ flask. Ethanethioamide ( $2.25 \mathrm{~g}, 0.03 \mathrm{~mol}, 3 \mathrm{M}$ solution in ethanol) was added in $3 \mathrm{~h}$ under vigorous stirring. The reaction mixture was stirred for additional $12 \mathrm{~h}$, and the precipitation was isolated with centrifugation and washed with ethanol for 5 times. The received PVBC@PbS nanospheres were dried in vacuum at $40^{\circ} \mathrm{C}$ for $24 \mathrm{~h}$.

\section{Removing the PVBC templates by calcination}

The PVBC@PbS core-shell hybrid nanospheres were added into quartz dish and then put into tubular-furnace, after vacuum pumping and circulated by argon. The temperature was raised to $600^{\circ} \mathrm{C}$ and stood for $6 \mathrm{~h}$, followed by cooling to room temperature. The nanospheres were dispersed into $50 \mathrm{~mL}$ THF in a $100 \mathrm{~mL}$ flask and stirred for $12 \mathrm{~h}$. The mixture was filtered and washed with deionized water for 3 times. The received hollow $\mathrm{PbS}$ nanospheres were dried in vacuum at $40^{\circ} \mathrm{C}$ for $24 \mathrm{~h}$.

\section{Release behavior of hollow PbS nanospheres loaded with phenol}

Due to the sensitive channel pathway in hollow $\mathrm{PbS}$ nanospheres, the controlled release behavior of hollow $\mathrm{PbS}$ nanospheres loaded with phenol was investigated. The hollow $\mathrm{PbS}$ nanospheres embedded with phenol was prepared by three different ways. First, $100 \mathrm{mg}$ of hollow $\mathrm{PbS}$ nanospheres was added into $20 \mathrm{~mL}$ phenol aqueous solution $(0.01 \mathrm{M})$. The hollow $\mathrm{PbS}$ nanospheres were then collected by centrifugation after incubation at room temperature for $24 \mathrm{~h}$ and washing by deionized water for 5 times. Second, hollow $\mathrm{PbS}$ nanospheres (100 mg) were heated to $300^{\circ} \mathrm{C}$ under nitrogen and then added into $20 \mathrm{~mL}$ phenol aqueous solution $(0.01 \mathrm{M})$ directly. The nanospheres were then collected after stirring for $30 \mathrm{~min}$ and washed by deionized water for 5 times. Third, hollow $\mathrm{PbS}$ nanospheres $(100 \mathrm{mg})$ were added into $20 \mathrm{~mL}$ phenol aqueous solution $(0.01 \mathrm{M})$ and stirred for $3 \mathrm{~h}$ under vacuum at room temperature. The nanospheres were collected after washing by deionized water for 5 times. The absorbance of the solution was measured by the UV spectrophotometer, and the phenol concentration was calculated by the use of the standard curves.

The hollow $\mathrm{PbS}$ nanospheres embedded with phenol were added into dialysis bags, followed by the soaking in $50 \mathrm{~mL}$ deionized water under stirring. The solution was measured by the UV spectrophotometer at wavelength of $268 \mathrm{~nm}$. After $36 \mathrm{~h}$, the data were collected and 


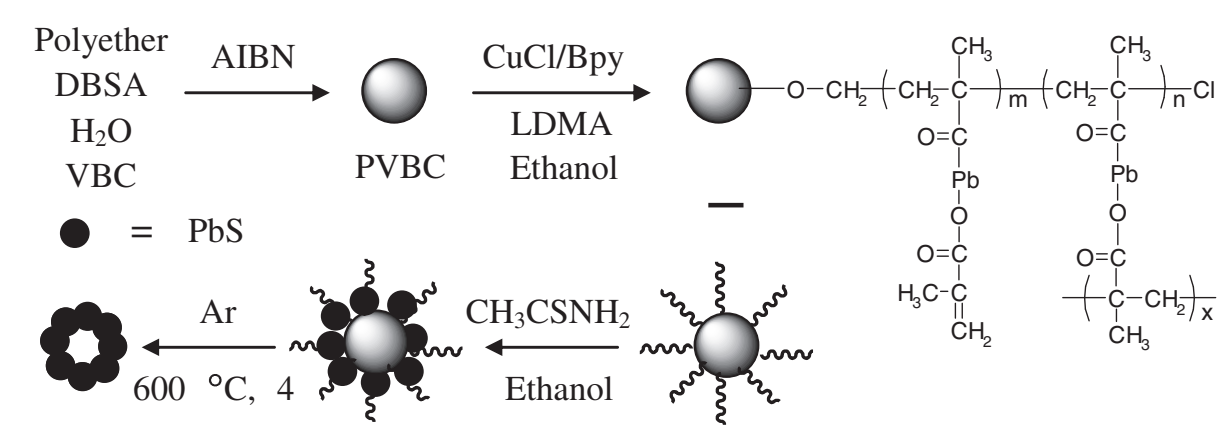

Scheme 1 The synthetic process for the preparation of hollow PbS nanospheres with sensitive channel pathway in shell.

analyzed in comparison with the release behavior of pristine phenol.

\section{Characterization}

The fourier transform infrared resonance (FTIR) spectra of the samples dispersed in $\mathrm{KBr}$ disks were recorded on a SHIMADZU IRprestige-21 spectrophotometer. Transmission electron microscope (TEM) analysis was used to characterize the morphology of the nanospheres. In a typical experiment, several drops of the colloidal dispersion were introduced onto a carbon film supported by a copper grid. The droplet was allowed to dry in air, and then observed under a JEM-2010 (HR) transmission electron microscope operating at an acceleration voltage of $100 \mathrm{kV}$. The X-ray diffraction (XRD) study of the samples was carried out on a Bruker D8 Focus X-ray diffractometer, operating at $40 \mathrm{kV}$ and $40 \mathrm{~mA}$ with a copper target $(\lambda=1.54 \AA)$ and at a scanning rate of $2^{\circ} / \mathrm{min}$. The ultraviolet-visible spectra of the samples were obtained with a Hitachi UV-2300 spectrophotometer. The photoluminescence (PL) of the samples was measured on a Shimadzu RF-5301PC spectrofluorophotometer. Both experiments were performed at the ambient temperature.

\section{Results and discussion}

The synthetic process of hollow $\mathrm{PbS}$ nanospheres was illustrated in Scheme 1. The PVBC latex nanoparticles were obtained by the radical polymerization of VBC initiated by the AIBN in the presence of surfactants. Then, the chloro atoms on the surfaces of PVBC nanoparticles initiated the ATRP of LDMA to afford the PVBC-g -PLDMA. The obtained PVBC-g-PLDMA nanospheres were then reacted with ethanethioamide, leading to the formation of PVBC@PbS core-shell nanospheres. After removal of the PVBC templates by calcination, the hollow $\mathrm{PbS}$ nanospheres were then received. The corresponding release behavior of phenol adsorbed by hollow $\mathrm{PbS}$ nanospheres was investigated. It is noted that the distribution of benzyl chloride groups on the surfaces of PVBC latex nanoparticles was not homogeneous, which could lead to the formation of PLDMA with different densities. After the reaction, the $\mathrm{PbS}$ nanoparticles with different diameters and densities were distributing on the surfaces of the PVBC latex nanoparticles, leading to the formation of $\mathrm{PbS}$ shell by removal of the inner PVBC matrix.

\section{Structure analysis}

Figure 1 shows the IR spectra of the specimens. For the pristine PVBC, the peaks at 1610 and $1500 \mathrm{~cm}^{-1}$ were ascribed to the absorption of benzene ring. The peaks at 3100, 2950, 2860 and $1380 \mathrm{~cm}^{-1}$ were attributed to $\mathrm{C}-\mathrm{H}$ stretching vibrations of benzene ring, aliphatic segments, and $\mathrm{C}-\mathrm{H}$ shear vibration, respectively. Furthermore, the absorption peak at $1265 \mathrm{~cm}^{-1}$ corresponded to the $-\mathrm{CH}_{2} \mathrm{Cl}$ group. It is indicated that the PVBC has been successfully obtained during the reaction process. For the

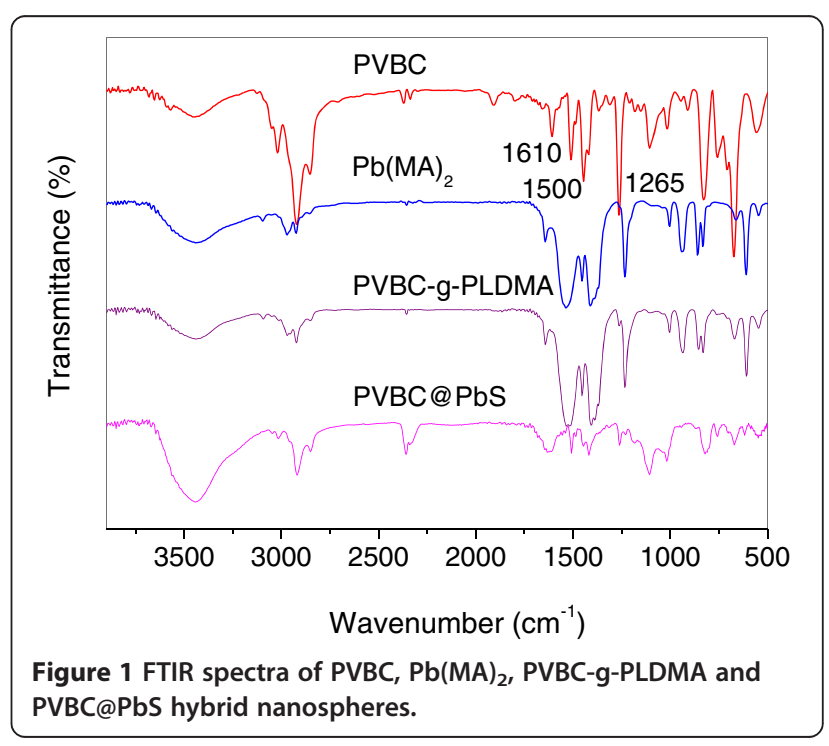




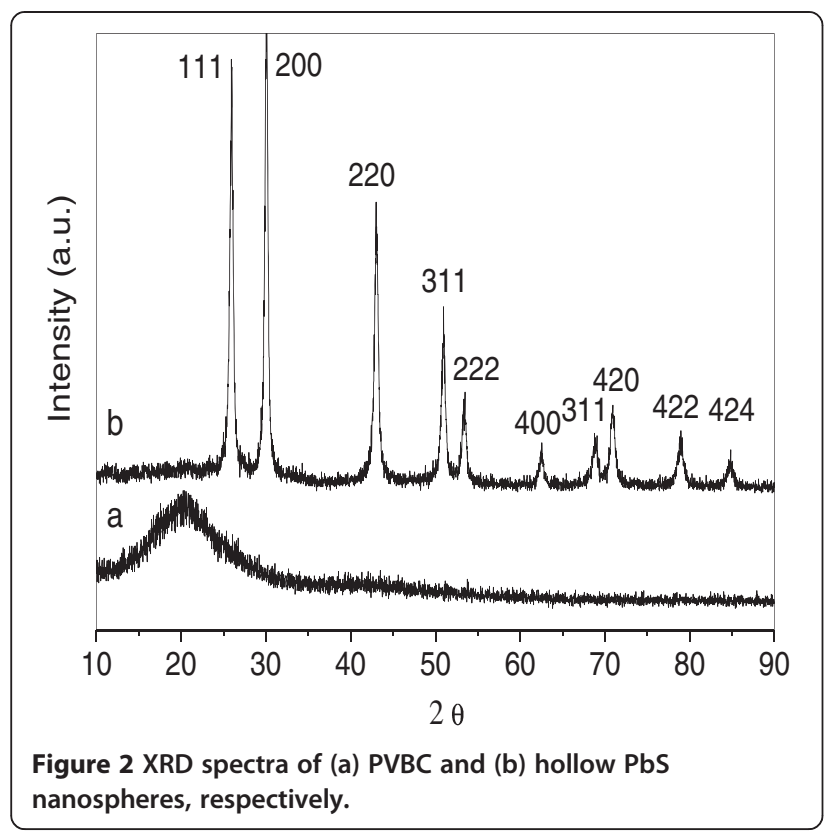

$\mathrm{Pb}(\mathrm{MA})_{2}$ monomer, the peaks at 1515 and $1450 \mathrm{~cm}^{-1}$ corresponded to the asymmetric carboxylate vibration, and the peak at $1387 \mathrm{~cm}^{-1}$ was ascribed to the symmetric carboxylate vibration. After the grafting of PLDMA onto PVBC, the characteristic absorption peaks attributed to both of PVBC and $\mathrm{Pb}(\mathrm{MA})_{2}$ were observed. By the treatment of ethanethioamide, the relative intensity of absorption peaks at $1520 \mathrm{~cm}^{-1}$ significantly decreased, due to the formation of $\mathrm{PbS}$ during the reaction. Thus, the amount of carboxylate groups decreased, leading to the decrease of the relative intensity of the absorption peaks at $1520 \mathrm{~cm}^{-1}$. The PVBC@PbS nanospheres were successfully obtained after the reaction process.

The X-ray spectrum was further performed to investigate the crystalline structure of PVBC and hollow $\mathrm{PbS}$ nanospheres. As shown in Figure 2, the PVBC nanospheres exhibited a broad reflection peak at $2 \theta=20.0^{\circ}$, indicating that PVBC was amorphous. In contrast, the hollow PbS nanospheres showed sharp reflection peaks, indicating that the nanospheres were highly crystalline. The strong diffraction peaks corresponded to the face-centered cubic of $\mathrm{PbS}$ (111), (200), (220), (311), (222), (400), (331) crystal face consistent with powder diffraction card (PDF No.5-0592). The above results indicate that the $\mathrm{PbS}$ nanospheres have been successfully prepared. Based on the Scherrer equation, the diameter of the hollow $\mathrm{PbS}$ nanospheres was calculated to be about $15.0 \mathrm{~nm}$.

\section{Morphology}

Both of SEM and TEM analysis were used to observe the morphology of hollow PbS nanospheres. As shown in Figure $3 \mathrm{a}$, the broken $\mathrm{PbS}$ nanospheres could be observed. The diameter of the nanospheres ranged from 80 to $110 \mathrm{~nm}$, and the average size was determined to be about $100 \mathrm{~nm}$. The thickness of the shell was about $20 \mathrm{~nm}$. Furthermore, in Figure 3b, the color depth of grain of the nanospheres was inconsistent, indicating of the hollow structure in nanospheres. In addition, some of the nanospheres exhibited peanut-shaped hollow structure, which might be due to the adjacent nanospheres collided together during the reaction process. It is concluded that the hollow $\mathrm{PbS}$ nanospheres were obtained.

\section{Optical properties}

Figure 4 shows the typical reflectance UV-vis spectra for hollow PbS nanospheres. The absorption edge started from the near infrared region, and the absorption region was about $1800 \mathrm{~nm}$ (band gap $0.69 \mathrm{eV}$ ), showing of an obvious blue shift in contrast to absorption region at $3200 \mathrm{~nm}$ for PbS (band gap $0.42 \mathrm{eV}$ ). It is indicated that the significant quantum size effect could be noticed. In the photoluminescence spectra (Figure 5), the hollow $\mathrm{PbS}$ nanospheres emitted at $872 \mathrm{~nm}$ when excited at $580 \mathrm{~nm}$. Nano-sized semiconductor particles generally exhibited a
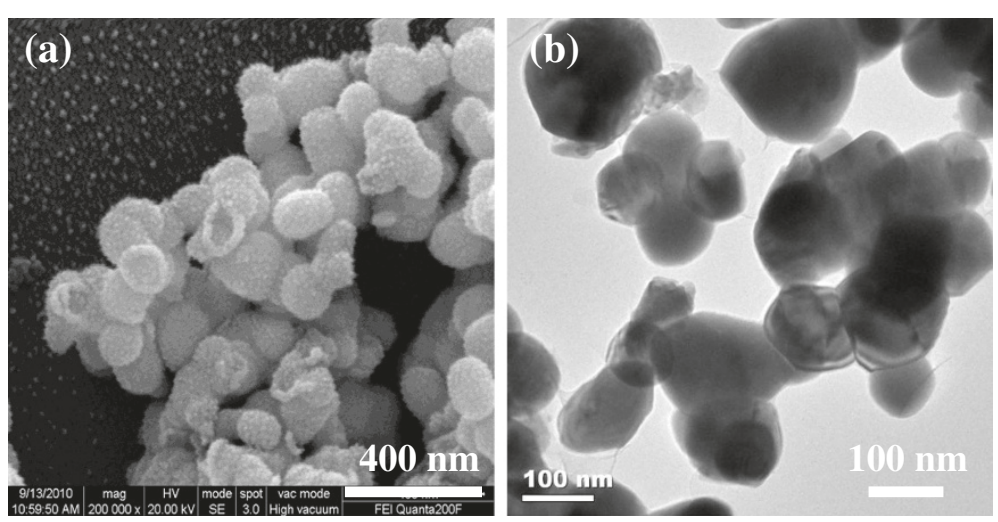

Figure 3 (a) SEM and (b) TEM graphs of hollow PbS nanospheres, respectively. 


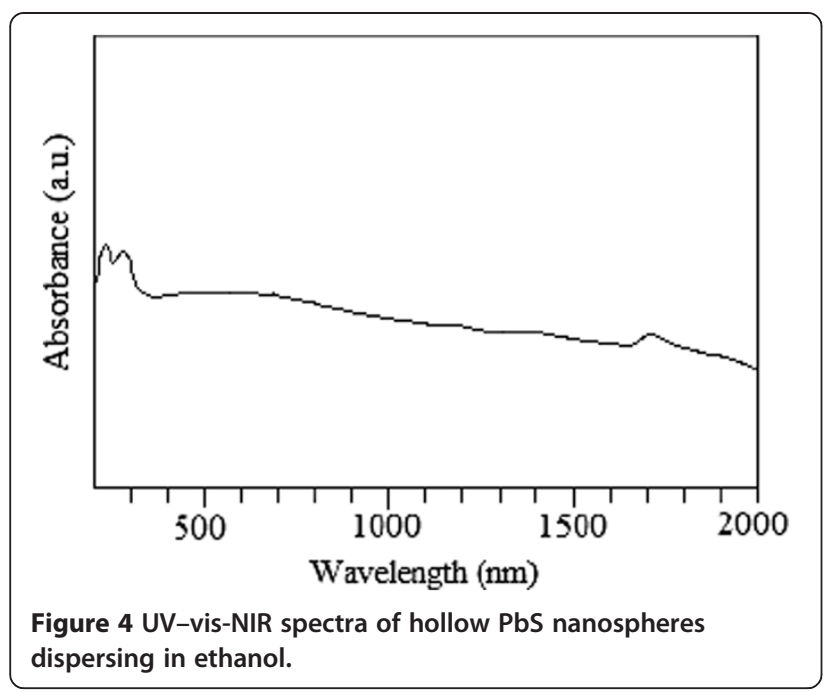

threshold energy in the optical absorption measurements, due to the size-specific band gap structures, which could be affected by the blue shifting of the absorption edge with decreasing particle size. This provided an indirect way to evaluate, at least quantitatively, the variation of particle core dimensions. Therefore, it is concluded that the hollow $\mathrm{PbS}$ nanospheres exhibited a rather small size, which was consistent with the results in SEM and TEM observation. The UV-vis absorption edge showed a very significant blue shift from the bulk $\mathrm{PbS}$ crystals, indicating the quantum confinement.

\section{Release behavior of phenol adsorbed by hollow $\mathrm{PbS}$ nanospheres}

The standard curve between concentration and absorbance (determined by UV spectra) for the phenol aqueous solution has been carefully prepared. It is revealed

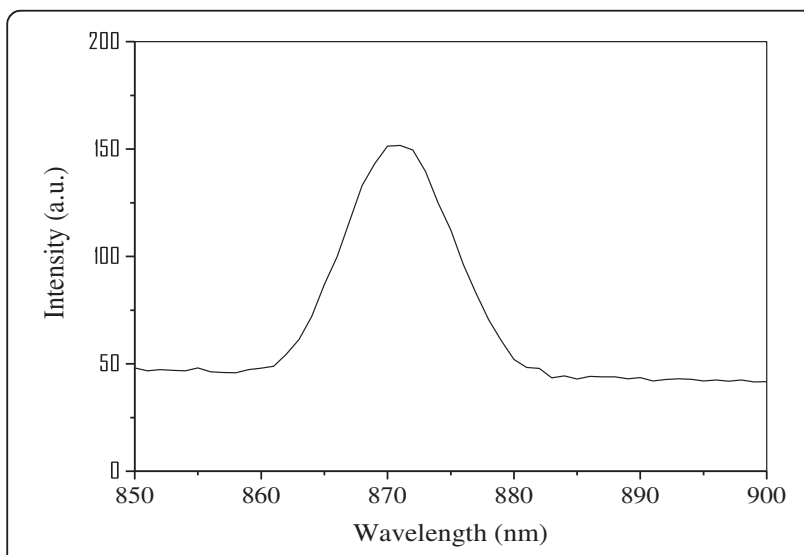

Figure 5 Photoluminescence spectrum of the hollow $\mathrm{PbS}$ nanospheres in ethanol excited at $872 \mathrm{~nm}$. that a good linear relationship could be obtained at the phenol concentration below $30 \mathrm{mg} / \mathrm{L}$. Based on the standard curve, the phenol concentration in solution could then be calculated after the measurement of absorbance in solution.

The quality of phenol adsorbed by the hollow $\mathrm{PbS}$ nanospheres was dependent on the preparation conditions. The percent of loaded phenol encapsulated by hollow $\mathrm{PbS}$ nanospheres was $61.9 \%, 60.8 \%$ and $76.3 \%$ for the specimens prepared at room temperature, quenching and vacuum conditions, respectively. The sample after treating by the vacuum absorbed more content of phenol than the other methods. It is suggested that the phenol could penetrate into the hollow nanospheres via the shell. Figure 6 shows the release behavior of phenol adsorbed by hollow $\mathrm{PbS}$ nanospheres which were prepared at different conditions. For the pristine phenol, about 35\% phenol released from the dialysis bag after $3 \mathrm{~h}$, and $80 \%$ phenol released after $5 \mathrm{~h}$. In comparison, the hollow $\mathrm{PbS}$ nanospheres released the phenol in a much lower rate. About $50 \%$ of the phenol released from the dialysis bag after $8 \mathrm{~h}$. It is noticed that the phenol release rate was almost constant and exhibited a controlled releasing behavior. Almost 95\% phenol released after $36 \mathrm{~h}$, and the releasing time was much longer than that of pristine phenol. Therefore, the hollow $\mathrm{PbS}$ nanospheres could potentially be used for the controlled release of drugs.

\section{Conclusions}

The hollow PbS nanospheres have been successfully fabricated via the convenient template method, including the preparation of PVBC latex nanoparticles by radical polymerization, grafting of PLDMA onto PVBC via ATRP,

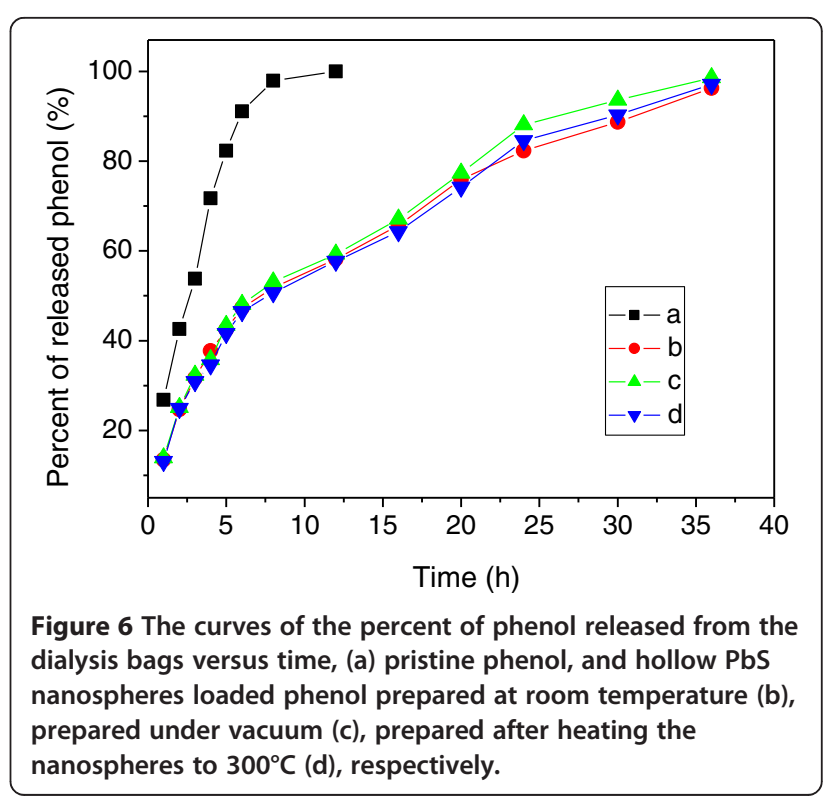


and removal of the polymer template by calcination. The hollow $\mathrm{PbS}$ nanospheres were highly crystalline and exhibited the quantum confinement due to the smaller average size of $\mathrm{PbS}$ nanospheres than that of the excitonic Bohr radius of the bulk PbS. The hollow $\mathrm{PbS}$ nanospheres could be used for the controlled release of phenol, which might be contributing to the existence of PbS nanosphere shell.

\section{Competing interests}

The authors declared that intellectual property right of the article belonged to Bengbu College.

\section{Authors' contributions}

JY carried out the design and most of the experimental work including the synthesis of the latex nanoparticles and preparation of hollow lead sulfide (PbS) nanospheres in this article. In addition, he analyzed the data carefully and drafted the manuscript. LS carried out the FTIR, UV and PL analysis. SG carried out the XRD, TEM and SEM analysis. All authors read and approved the final manuscript.

\section{Acknowledgements}

This Project Supported by Scientific Research Fund of Anhui Provincial Education Department (KJ2010B104)

Received: 27 January 2013 Accepted: 25 April 2013

Published: 18 July 2013

\section{References}

Asunskis DJ, Bolotin IL, Hanley L (2008) Nonlinear optical properties of PbS nanocrystals grown in polymer solutions. J Phys Chem C 112:9555-9558

Caruso DF (2000) Hollow capsule processing through colloidal templating and self-assembly. Chem A Eur J 6:413-419

Chen TY, Du BY, Fan ZQ (2012) Facile fabrication of polymer nanocapsules with cross-linked organic-inorganic hybrid walls. Langmuir 28:11225-11231

Dave AM (1984) Synthesis of lead dimethacrylate. Polymer 25:1020-1022

Deng DW, Zhang WH, Chen XY, Liu F, Zhang J, Gu YQ, Hong JM (2009) Facile synthesis of high-quality, water-soluble, near-infrared-emitting PbS quantum dots. Eur Polym I Inorg Chem 23:3440-3446

Ding YH, Liu XX, Guo R (2007) Synthesis of hollow PbS nanospheres in plutonic F127/cyclohexane/ $\mathrm{H}_{2} \mathrm{O}$ microemulsions. Colloids Surf A Physicochem Eng Aspects 296:8-18

Du BY, Cao Z, Li ZB, Mei AX, Zhang XH, Nie JJ, Xu JT, Fan ZQ (2009) One-pot preparation of hollow silica spheres by using thermosensitive poly $(\mathrm{N}$ isopropylacrylamide) as a reversible template. Langmuir 25:12367-12373

Gao JB, Luther JM, Semonin OE, Ellingson RJ, Nozik AJ, Beard MC, Wang Y, Xia CY (2005) Monodisperse spherical colloids of Pb and their use as chemical templates to produce hollow particles. Adv Mater 17:473-477

Gao JB, Luther JM, Semonin OE, Ellingson RJ, Nozik AJ, Beard MC (2011) Quantum dot size dependent J-V characteristics in heterojunction ZnO/PbS quantum dot solar cells. Nano Lett 11:1002-1008

Huang HY, Remsen EE, Kowalewski T, Wooley KL (1999) Nanocages derived from shell cross-linked micelle templates. J Am Chem Soc 121:3805-3806

Jain PK, Amirav L, Aloni S, Alivisatos AP (2010) Nanoheterostructure cation exchange: Anionic framework conservation. J Am Chem Soc 132:9997-9999

Lau YKA, Chernak DJ, Bierman MJ, Jin S (2009) Formation of PbS nanowire pine trees driven by screw dislocations. J Am Chem Soc 131:16461-16471

Lee SM, Jun YW, Cho SN, Cheon JW (2002) Single-crystalline star-shaped nanocrystals and their evolution: Programming the geometry of nanobuilding blocks. J Am Chem Soc 124:11244-11245

Lee JS, Shevchenko EV, Talapin DV (2008) Au-PbS core-shell nanocrystals: Plasmonic absorption enhancement and electrical doping via intra-particle charge transfer. J Am Chem Soc 130:9673-9675

Machol JL, Wise FW, Patel RC (1993) Vibronic quantum beats in PbS microcrystallites. Phys Rev B 48:2819-2822
Matyjaszewski K, Miller PJ, Shukla N, Immaraporn B, Gelman A, Luokala BB, Siclovan TM, Kickelbick G, Vallant T, Hoffmann H, Pakula T (1999) Polymers at Interfaces: Using atom transfer radical polymerization in the controlled growth of homopolymers and block copolymers from silicon surfaces in the absence of untethered sacrificial initiator. Macromolecules 32:8716-8724

McDonald SA, Konstantatos G, Zhang S, Cyr PW, Klem EJD, Levina L, Sargent EH (2005) Solution-processed PbS quantum dot infrared photodetectors and photovoltaics. Nat Mater 4:138-142

Noone KM, Strein E, Anderson NC, Wu PT, Jenekhe SA, Ginger (2010) Broadband absorbing bulk heterojunction photovoltaics using low-bandgap solutionprocessed quantum dots. Nano Lett 10:2635-2639

Peng ZP, Jiang YS, Song YH, Wang C, Zhang HJ (2008) Morphology control of nanoscale PbS particles in a polyol process. Chem Mater 20:3153-3162

Ridley BA, Nivi B, Jacobson JM (1999) All-inorganic field effect transistors fabricated by printing. Science $286: 746-749$

Wang SH, Yang SH (2000) Preparation and characterization of oriented PbS crystalline nanorods in polymer films. Langmuir 16:389-397

Wang SF, Gu F, Lü MK (2006) Sonochemical synthesis of hollow PbS nanospheres. Langmuir 22:398-401

Zhou GJ, Lü MK, Xiu ZL, Wang SF, Zhang HP, Zhou YY, Wang SM (2006) Controlled synthesis of high-quality PbS star-shaped dendrites, multipods, truncated nanocubes, and nanocubes and their shape evolution process. J Phys Chem B 110:6543-6548

doi:10.1186/2193-1801-2-323

Cite this article as: Ye et al:: Fabrication of hollow PbS nanospheres and application in phenol release. SpringerPlus 2013 2:323.

\section{Submit your manuscript to a SpringerOpen ${ }^{\circ}$ journal and benefit from:}

- Convenient online submission

- Rigorous peer review

- Immediate publication on acceptance

- Open access: articles freely available online

- High visibility within the field

- Retaining the copyright to your article

Submit your next manuscript at $\gg$ springeropen.com 